\title{
Optimization of lon Source Head Position in the Central Region of DECY-13 Cyclotron
}

\author{
Silakhuddin ${ }^{*}$ and I.A. Kudus \\ Center for Accelerator Science and Technology, National Nuclear Energy Agency, \\ Jl. Babarsari, Yogyakarta 55281, Indonesia
}

\section{ARTICLE INFO}

\section{Article history:}

Received 09 November 2016

Received in revised form 08 March 2017

Accepted 20 April 2017

\section{Keywords:}

Cyclotron

Ion source

Head position

Central region

Simulation

Optimization

\begin{abstract}
A B S T R A C T
Optimization of the ion source head position of the DECY-13 Cyclotron in the central region has been carried out based on simulation process using a particle tracking program written in Scilab 5.2.1. The simulated particle was the $\mathrm{H}^{-}$ion that was accelerated in DECY-13 Cyclotron. The input for the program were the magnetic field and the electric field in the central region that were calculated by Opera-3D software package and TOSCA module. The optimized position of ion source head position is in a radius of $2 \mathrm{~cm}$ relative to the zero point of the magnet and at a distance of $4 \mathrm{~mm}$ relative to the puller. This result can be useful for determining the configuration of the parts in the central region when it is tested for generating the first ion beam in the future.
\end{abstract}

(C) 2017 Atom Indonesia. All rights reserved

\section{INTRODUCTION}

The operation of a cyclotron facility, especially a commercial cyclotron, requires the maximization of the existing ion beam $\mathrm{c}$ urrent by optimization of the operating param eters. The parameters optimized are either fixed or variable parameters. A maximized beam current can be obtained if the ion extraction process from the ion source slit and the acceleration process are perfect. In this case, the beam loss in the extraction and acceleration processes are minimized. The minimum beam loss gives two advantages: operating cost efficiency and radiation reduction in the cyclotron chamber.

The ions that are produced inappropriately, or are in inappropriate conditions, can cause coherent oscillations thath result in deteriorated acceleration and beam loss during acceleration process. Therefore, optimization of the initial electric and magnetic fields was performed to determine the starting position of the ions and the geometry and position of the puller that pulls the ions from the chimney of the ion source [1]. The optimization can be carried out by looking for a correct position of the ion source head relative to the puller before

\footnotetext{
* Corresponding author.

E-mail address: silakh@batan.go.id

DOI: https://doi.org/10.17146/aij.2017.584
}

minimizing the problem with the acceleration process. The beam loss in the acceleration process is caused by three problems: collisions between ion beam and residual gas molecules, collision between ion beam and cyclotron components, and collisions between beam paths themselves. The first problem is caused by an insufficiently high vacuum, and a calculation that has already been done in the ARRONAX Cyclon 70 cyclotron shows that a vacuum level of $1 \times 10^{-6}$ Torr is needed if the targeted maximum beam loss is about $10 \%$ [2]. The second problem comes from the vertical oscillation of ion beams hitting dee components and ion source arms; it can be overcome by adjustment of vertical gap of the center block [1]. The third problem comes from a radial oscillation that is caused by the insufficient accuracy of the gap between the head slit of the ion source and the puller. The coherent radial oscillations result in reduced acceleration and increased beam loss before extraction. Therefore, the geometry and position of the puller that pulls the ions from the chimney of the ion source head has to be optimized.

From the above description, it can be summarized that the problems caused by the inaccuracy of horizontal head-puller are: the particle beam does not pass extraction, and the particle beam's failure to pass the initial acceleration because of collisions between the tracks themselves 
due to radial oscillations in the central region.

The development of the DECY-13 cyclotron at PSTA-BATAN is now in the components construction phase and will continue with the installation of the ion source in central region of the cyclotron [3]. As in the general installation of the ion source in a small cyclotron, a PIG ion source is placed internally to produce $\mathrm{H}^{-}$ions [4-6]. Simulations that examine patterns of the beam path in the central region have also been performed [7], but it has not been optimized by means of varying the width of the head-puller gap to obtain the high efficiency of the ion beam extraction and initial ion beam acceleration processes. This paper presents the results of the simulations on the effects of position variation of the ion source head to the qualitative efficiencies of both processes.

The optimization is based on the patterns of the ion beam trajectory during the extraction and initial acceleration using a single particle trajectory tracker program in a cyclotron. The results of these simulations will be used to determine an approximate gap width between the ion source head and the puller to be used when the ion source is tested in the cyclotron for ion beam production in the future.

\section{EXPERIMENTAL METHODS}

\section{Simulation object}

The object of the simulation is the central region of the DECY-13 cyclotron that is presently under construction in PSTA-BATAN, Yogyakarta. The cyclotron system in which the ion source is installed uses a dee system that operates in a pushpush mode so that the middle of both dees are connected to each other. In the middle of the dee, a puller is attached to pull negative ions from the ion source slit. The positions of the ion source and the puller are not at the center of the cyclotron, but on a radius of the initial circle of the beam. The geometry of the central region of the cyclotron is shown in Fig. 1.

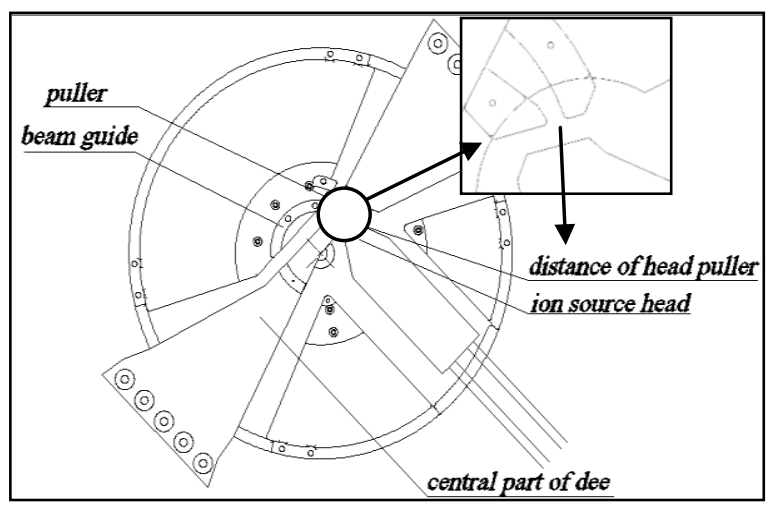

Fig. 1. Scheme of DECY-13 cyclotron's central region.
The puller is attached to the central dee at the $40-\mathrm{kV}$ peak voltage of the accelerating radiofrequency (RF) on a fixed position. The canal of the puller is $2 \mathrm{~mm}$ wide and $12 \mathrm{~mm}$ long. The distance from the ion source head to the puller can be adjusted by moving the ion source head to adjust the forward-backward distance or gap. The head can also be moved laterally to determine right-left position.

\section{Steps of simulations}

The steps of the simulations of ion trajectories are shown in the flowchart in Fig. 2.



Fig 2. Flowchart of optimization process.

\section{Determining the initial position of the ion source}

The initial position of the ion source head needs to be determined first before optimization. The position includes the radial position relative to the central point of the cyclotron and the initial gap, i.e. the maximum gap as a reference of the head-puller distance. As a rough approximation using a simple and well known formulae, the radial position of the ion source head from the cyclotron center point is as given by eq. (1):

$$
r=\frac{1}{B} \sqrt{\frac{2 m V}{q}}
$$


where $B$ is the magnetic field (in T) of cyclotron in the central area, $q$ is charge (in $\mathrm{C}$ ) of $\mathrm{H}^{-}$ions, $m$ is the mass (in $\mathrm{kg}$ ) of the $\mathrm{H}^{-}$ion, and $V$ is the effective voltage (in $\mathrm{V}$ ) of puller or dee (i.e., peak voltage divided by 1.4). The initial gap (forward-backward distance) is determined by finding the maximum distance at which an $\mathrm{H}^{-}$ion passing from the anode slit traverses up to the front end of the puller, i.e., a path starting at the zero phase of the electric field of puller and finishing at the phase of $180^{\circ}$ within a half-period of the RF phase. So, the distance $d$ (in $\mathrm{m}$ ) is as given by eq. (2):

$$
d=\frac{1}{2} a t^{2}=\frac{1}{2} \frac{F}{m}\left(\frac{T}{2}\right)^{2}=\frac{1}{2} \frac{q V}{d m}\left(\frac{1}{2 f}\right)^{2}
$$

and then

$$
d=\frac{1}{2 f} \sqrt{\frac{1}{2} \frac{q V}{m}}
$$

where $a$ is the acceleration (in $\mathrm{m} / \mathrm{s}^{2}$ ) of the ions, $t$ is the travel time (in s) of a particle from the anode slit to the puller (= half of $\operatorname{rf}$ period), $F$ is the electric force (in N) of the puller to the ion, $m$ is ionic mass (in $\mathrm{kg}$ ), $T$ is the period (in s) of the voltage of the puller, $q$ is the ionic charge (in C), $V$ is the effective voltage (in $\mathrm{V}$ ) of dee or puller, and $f$ is the RF frequency (in $\mathrm{Hz}$ ) of accelerating electric field.

\section{Cyclotron operation parameters}

The central region of the DECY-13 cyclotron had the following parameters: dee effective voltage of $28 \mathrm{kV}$ (peak voltage is $40 \mathrm{kV}$ ), RF frequency of $77.66 \mathrm{Mhz}$, and magnetic field at the center of $1.275 \mathrm{~T}$.

\section{Computing electric and magnetic fields}

It is difficult to analytically calculate the electric field and magnetic field distribution at any point in the cyclotron, but it can be done by numerical methods with the Opera 3D software and Tosca modules $[8,9]$. The values of electric field and magnetic field were imported from a map data created by using Opera-3D software and TOSCA modules that had been prepared in advance [10].

\section{Changing the ion source position}

The change of ion source position is for finding a desired ion trajectory that reflects the optimum position of the ion source head.
The desired ion trajectory is a trajectory that passes the central extraction canal of the puller without touching the walls and then the trajectory can undergo several preliminary rounds without touching other trajectories or the components of the central region.

Having obtained the reference point, i.e., the radial position of the puller and the head-puller initial distance, the next step was finding the range of forward-backward positions and right-left positions of the head relative to the puller around the reference point. The determination was performed by varying two kinds of positions (forward-backward and right-left) with the initial phase of zero degree, and for every position the $\mathrm{H}^{-}$ trajectory was observed by simulation using a program of Scilab 5.4.1. This program employed a Runge-Kutta numerical algorithm. This method was also followed in Korea for a cyclotron design by using the pwheel program [3]. The value of the mínimum radial distance was limited to $3 \mathrm{~mm}$ because at the distances smaller than the limit electrical sparks could occur between the head and the puller. The variation process of the position will be stopped when the desired trajectory as mentioned above is achieved. The optimized ion source (i.e., head) position will be validated by simulating trajectory of positive hydrogen ion and the trajectory must be impossible to form a circular path as for the negative ions and must be restrained by a barrier, i.e., a puller or a beam guide.

\section{RESULTS AND DISCUSSION}

\section{Determination of initial position}

By using eq. (1) and by applying $B=1.25 \mathrm{~T}$, $m=1.67 \times 10^{-27} \mathrm{~kg}, V=28 \mathrm{kV}$, and $q=1.6 \times 10^{-19}$ $\mathrm{C}$, it was obtained that the radius of anode position was $0.02 \mathrm{~m}=2 \mathrm{~cm}$. The initial head-puller distance was obtained using eq. (3) and for $f=78 \mathrm{MHz}$, the result was $0.0077 \mathrm{~m}=7.7 \mathrm{~mm}$. Therefore, it was found that the range of observation of the initial position of the ion source at a radius of $2 \mathrm{~cm}$ and at the maximum distance between the anode and the puller was $7.7 \mathrm{~mm}$.

\section{Finding the optimum forward-backward position}

For distances of head relative to the puller of under $7 \mathrm{~mm}$, the simulated trajectories for several rounds of the $\mathrm{H}^{-}$path are shown in Fig. 3. The pictures show the $\mathrm{H}^{-}$trajectories for $d=3 \mathrm{~mm}$, $4 \mathrm{~mm}, 5 \mathrm{~mm}, 6 \mathrm{~mm}$, and $7 \mathrm{~mm}$, respectively. 

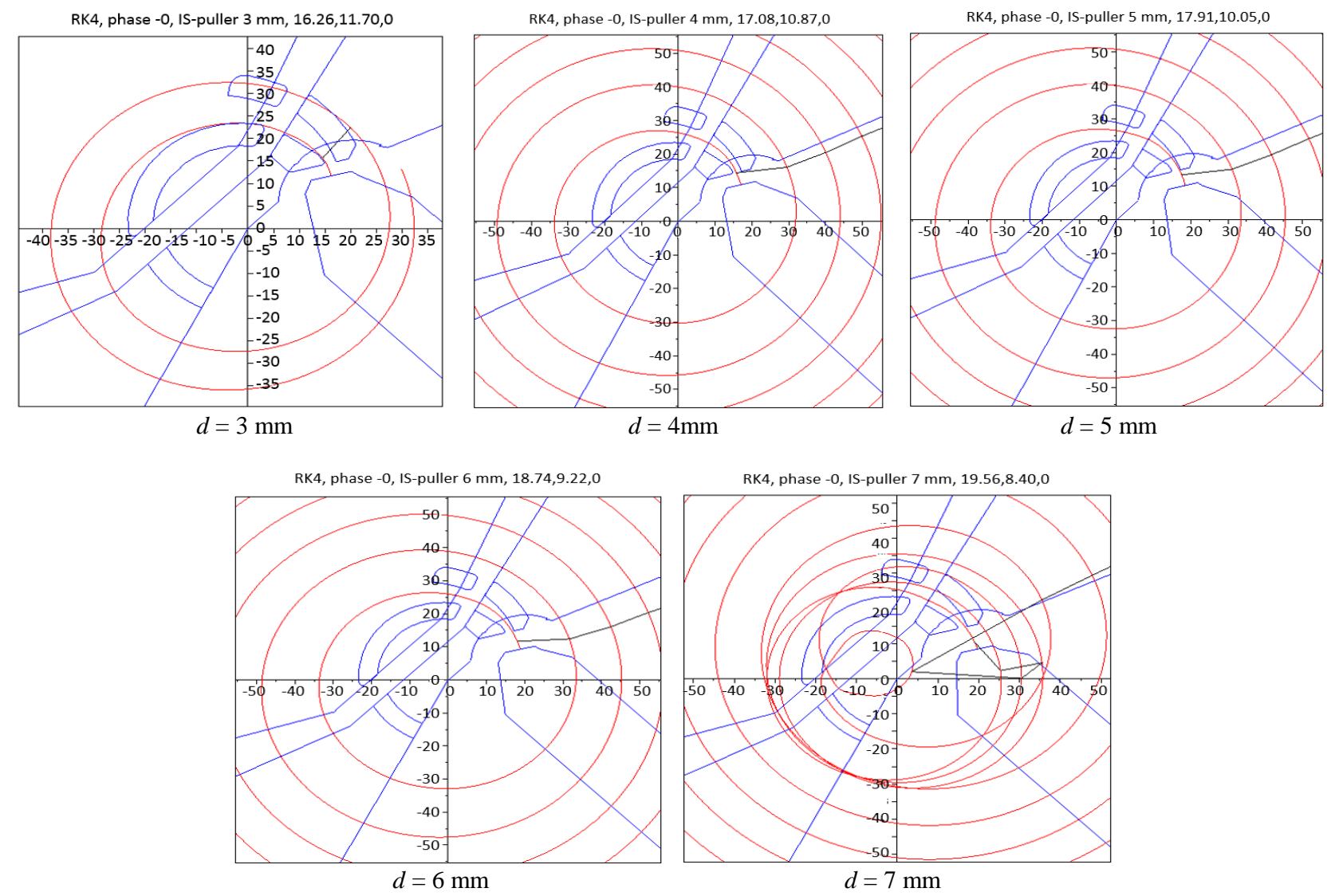

Fig. 3. Early trajectories of the $\mathrm{H}^{-}$ion in the central region for various radial positions $(d)$ of the head relative to the puller. The horizontal lines are $x$ axes and vertical lines are $y$ axes, all of those in $\mathrm{mm}$. The beam trajectories are indicated by red lines.
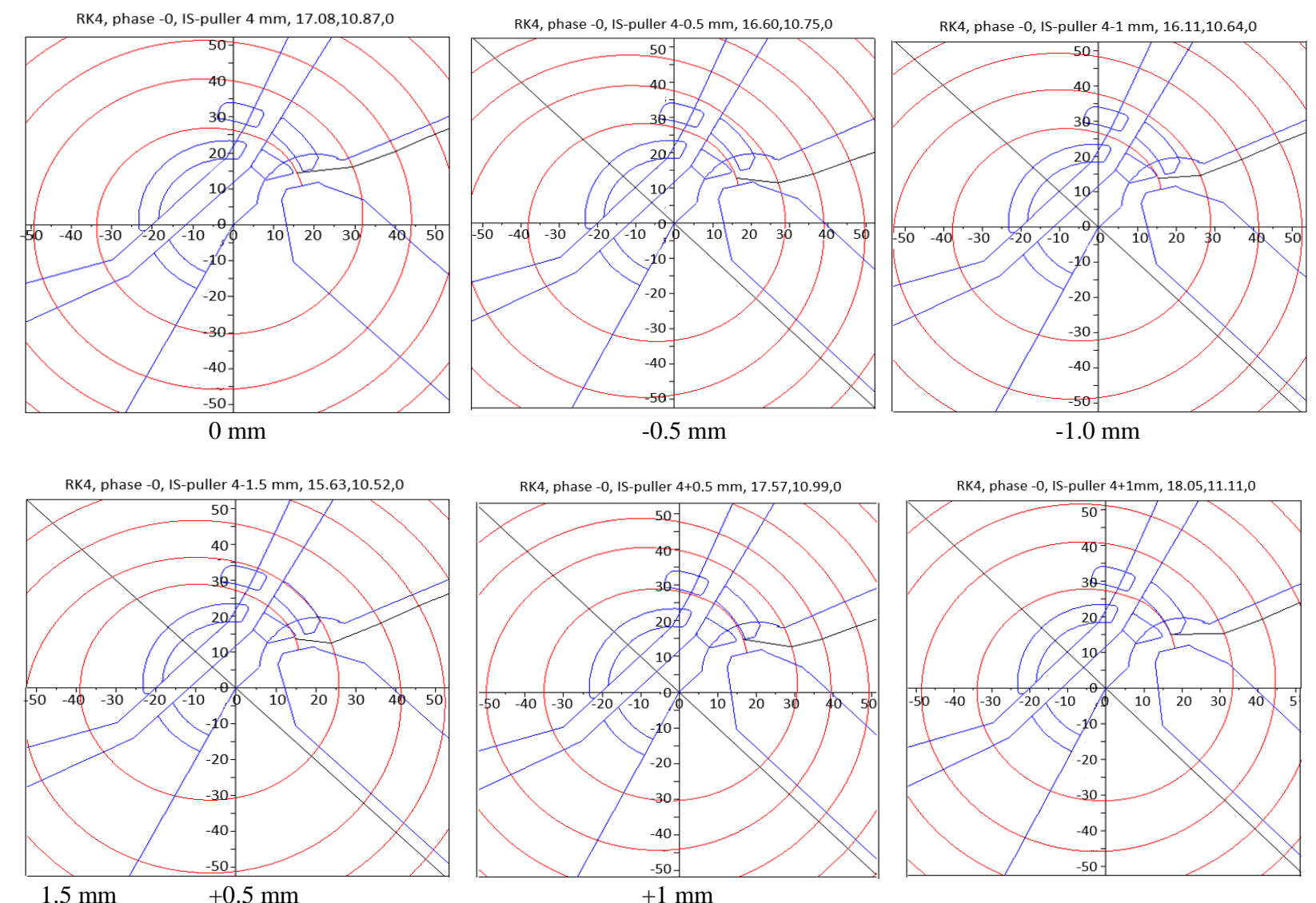

Fig. 4. Early trajectories of the $\mathrm{H}^{-}$ion in the central region for various azimuthal positions of the head relative to the puller. The horizontal lines are $x$ axes and vertical lines are $y$ axes, all of those in $\mathrm{mm}$. The beam trajectories are indicated by red lines. 
From these simulations it appears that the optimum distances that the ion can be extracted and accelerated in the central region were in the range of 4 to $6 \mathrm{~mm}$. At this distance, the ion passed the puller canal in the center without touching the wall of the canal. The most symmetrical trajectory occurred at a distance of $4 \mathrm{~mm}$. At a distance of under $4 \mathrm{~mm}$, the ion would touch the left side of the inner puller. This can be explained as follows. At a distance of less than $4 \mathrm{~mm}$, when the ion entered the puller, the ion was still being influenced by smaller electric field, which means the radius of the trajectory was still small so it touched the puller. At a gap of larger than $6 \mathrm{~mm}$, the ion struck the central region components after the second round; that may occur due to the delay of ion phase compared to RF dee phase, or in other words, the phase of the ion was no longer the same as the phase of the dee voltage.

\section{Finding the optimum right-left position}

Using $4 \mathrm{~mm}$ (the best forward-backward distance), the next step was simulating ion trajectories with variations on the right-left positions of the ion source head relative to the puller. Here, a position on the right original position is denoted by the + sign and on the left is by the - sign. The results of the beam trajectory simulations are shown in Fig. 4

By observing the results of those simulations, it appears that the positions where the ion beam can be extracted well ranged between -1 and $+0.5 \mathrm{~mm}$, or in the span of only $1.5 \mathrm{~mm}$ around the original point which is $0 \mathrm{~mm}$ position or exactly in the $2 \mathrm{~cm}$ radius.

Furthermore, this position will be used for observing the trajectory of $\mathrm{H}^{+}$ion and the result is shown in Fig. 5.



Fig. 5. Initial trajectory of the $\mathrm{H}^{+}$ion. The horizontal line is $x$ axis and vertical line is $y$ axis, all of those in $\mathrm{mm}$. The beam trajectory is indicated by red line.
The trajectory of the $\mathrm{H}^{+}$ion could not pass the puller canal and instead hits the near part of the puller. It means that the position that is assumed to be the best position does not allow the $\mathrm{H}^{+}$to have free trajectories. The last result also shows that the puller will receive the entire power of the $\mathrm{H}^{+}$beam, so the cooling treatment of the puller should be a concern.

\section{Comparison with other cyclotrons}

As it has been mentioned above, the best position of the ion source head is at a distance of $4 \mathrm{~mm}$ relative to the puller and at $2 \mathrm{~cm}$ radius. In this case, the RF frequency is $77.6 \mathrm{MHz}$ and the maximum puller/dee voltage is $40 \mathrm{kV}$. Table 1 shows the data of the puller-ion source head distances from several cyclotron facilities in connection with various RF frequencies.

Table 1. The distance puller-ion source head in several cyclotron facilities.

\begin{tabular}{llll}
\hline No. & \multicolumn{1}{c}{ Cyclotron } & RF frequency & $\begin{array}{c}\text { Puller-head } \\
\text { distance }\end{array}$ \\
\hline 1. & AMIT, Spain [11] & $60 \mathrm{MHz}$ & $6 \mathrm{~mm}$ \\
2. & High Intensity & $66.99 \mathrm{MHz}$ & $5 \mathrm{~mm}$ \\
3. & SuperConducting & $77.6 \mathrm{MHz}$ & $4 \mathrm{~mm}$ \\
& $\begin{array}{l}\text { Cyclotron, DTRA [12] } \\
\text { This paper }\end{array}$ & & \\
4. & KIRAMS 9 MeV [13] & $83 \mathrm{MHz}$ & $2.2 \mathrm{~mm}$ \\
\hline
\end{tabular}

In rough comparison, the result of this paper is logical because the lower accelerating radio frequency (as in the AMIT Cyclotron and DTRA's Superconducting Cyclotron) will produce slower ion movement, an therefore a farther distance is needed by the ions to arrive at the puller. On the contrary, a higher accelerating RF voltage frequency $(83 \mathrm{MHz}$ at KIRAMS $9 \mathrm{MeV}$ ) will produce faster ion movement, thus a smaller distance is sufficient.

\section{CONCLUSION}

The optimum position of the ion source head of DECY-13 Cyclotron has been obtained through the simulation of ion beam trajectory particle tracking using a software package written in Scilab 5.4.1. The best position of the ion source head is at $4 \mathrm{~mm}$ from the puller and on the $2-\mathrm{cm}$ radius. The position of the ion source head is also quite critical, it is derived from the fact that the viable distance between head and puller is only between 4 and $6 \mathrm{~mm}$ and the left-right span of just $1.5 \mathrm{~mm}$ in $2 \mathrm{~cm}$ radius. The comparison with other cyclotron facilities show that this result is quite 
in agreement, it means that it can be used to set the ion source position in the central región of the DECY-13 cyclotron.

\section{ACKNOWLEDGMENT}

This paper is made possible by the support by activity of research and development of DECY-13 Cyclotron at the Center for Accelerator Science and Technology. Therefore, the authors would like to thank the Head of the Center, the Head of Particle Physics section, and various other parties. The simulation program of ion beam trajectory was first created by Prof. Dr. Anggraita, and the authors also would like to thank him for his work.

\section{REFERENCES}

1. S.Y. Jung, H.W. Kim and M. Ghergherehchi, Journal of Instrumentation 9 (2014) 2.

2. V. Nuttens, M. Abs, J.L. Delvaux et al., Cyclotron Vacuum Model and H- Gas Stripping Losses, Proceedings of CYCLOTRONS 2010, Lanzhou, China (2010) 202.

3. S. Silakhuddin and S. Santosa, Atom Indonesia 41 (2015) 139.

4. J. Long, Z. Yang, P. Dong et al., Nuclear Science and Techniques 24 (2013) 040201.
5. M. Auger, S. Braccini, A. Ereditato et al., Meas. Sci. Technol. 26 (2015) 094006.

6. T. Yang, Z. Yang, P. Dong P. et al., Rev. Sci. Instrum. 83 (6) (2012) 063302

7. P. Anggraita, E. Mulyani and I.A. Kudus, Atom Indonesia 41 (2015) 145.

8. P. Anggraita, B. Santosa, Taufik et al., AIP Conf. Proc. 1454 (2012) 178.

9. J. Kang, B.H. Hong, D.H. An et al., IEEE Transactions on Applied of Superconductivity 22 (3) (2012) 4401104. doi:10.1109/TASC.2011. 2174567

10. Taufik, A. Hermanto, P. Anggraita et al., Atom Indonesia 40 (2014) 70.

11. C. Oliver, P. Abramian, B. Ahedo et al., Optimizing the Radioisotope Production with a Weak Focusing Compact Cyclotron, Proceedings of Cyclotrons2013, Vancouver, BC, Canada (2013) 430.

12. J.V. Minervini, High Intensity Superconducting Cyclotron, Technical Report Publishing of Defense Threat Reduction Agency USA, DTRA-TR-12-40 (2012).

13. X.J. Mu, M. Ghergherehchi, Y.H. Yeon et al., Chinese Physics C 38 (2014) 127004-5. 